\title{
VALIDATION OF DEEP FREEZING OF PILOT SAMPLES FOR CHECKING OF TIME STABILITY OF INDIRECT ANALYSES OF BASIC MILK COMPOSITION AND FOR THEIR LONG SHELF-LIFE
}

\author{
O. Hanuš, P. Hering, V. Genčurová, Z. Motyčka jun., R. Jedelská, J. Kopecký
}

Received: April 21, 2008

\begin{abstract}
HANUŠ, O., HERING, P., GENČUROVÁ, V., MOTYČKA, Z., JEDELSKÁ, R., KOPECKÝ, J.: Validation of deep freezing of pilot samples for checking of time stability of indirect analyses of basic milk composition and for their long shelf-life. Acta univ. agric. et silvic. Mendel. Brun., 2008, LVI, No. 5, pp. 57-68
\end{abstract}

Essential part of raw milk component measurement is indirect infraanalyse. The checking of time measurement stability is important there. The preparation of pilot samples was described. Information about deep frozen milk pilot sample stability are sporadic. Aim of this work was to verify the stability of long-term stored and deep frozen pilot samples (FPSs). Pilot samples were frozen in liquid nitrogen bath $-196^{\circ} \mathrm{C}$ and after that stored at $-21^{\circ} \mathrm{C}$ for one month (A) and more months (B) till analyse. The pilot samples were measured by CombiFoss 6000: fat F (\%); protein CP (\%); lactose L (\%); casein C (\%); solid non fat SNF (\%); urea U (mg/100ml); somatic cell count SCC (ths./ml). In the short-term (A) experiment the impact of freezing on pilot milk samples was relatively small, in the framework of acceptable value of repeatability $( \pm 0.02 \%)$ for components. The stability of values of FPSs was better according to standard deviations for important $\mathrm{F}$ evaluation in $\mathrm{H}$ (Holstein) breed samples. The repeatability of values of FPSs in the time was very good for other components. The repeatability was better for SCC in J (Jersey) breed samples ( \pm 16.5 thousands/ml). That is why the higher variability ( \pm 30.1 thousands $/ \mathrm{ml}$ ) in $\mathrm{H}$ sample is visible less sceptically. The apparent result trends were not observable in the repeatability development of important milk indicators in FPSs. The mild trend was only in CP, but this trend covered absolutely very small shift (variation range). There are only oscillations, which are similar between $\mathrm{J}$ and $\mathrm{H}$ samples. It is possible to attribute these oscillations rather to instrument effects than to sample effects. The oscilations were markedly higher in the long-term (B) experiment. Between $\mathrm{J}$ and $\mathrm{H}$ samples the oscilations were very similar in curves $\mathrm{F}$ and $\mathrm{L}$. It is also possible to attribute them more to instrumental effects. The repeatability values (standard deviations sd) of FPSs were mostly lower than $\pm 0.06 \%$. It is acceptable for result agreement between indirect and reference method. Relevant sd varied from $\pm 0.025 \%$ for L to $\pm 0.059 \%$ for F (in J breed) with exception $\mathrm{CP} \pm 0.085 \%$ in J sample. Also B procedure was shown as applicable for instrumental sability control in half year period. In practice the one reference value for concrete FPS is valid from one to next calibration. Persistent trend in repeatability of FPSs can indicate an inadequate instrumental drift. The applied FPS procedure is usable for stability control of instrumental measurement in milk laboratories.

milk, pilot sample, deep freezing, long shelf-life, measurement stability checking, indirect method, infraanalyse, fat, protein, somatic cell count

Essential part of raw milk component measurement is indirect analyse of samples on fat, protein and lactose contents by instruments which work on the basis of infrared spectrum measurement. It is important because of needs of milk recording for cattle genetical improvement purposes and also be- 
cause of milk quality determination for technological processing purposes. Because the infraanalyse works on indirect principle it is necessary to calibrate the instruments regularly.

In 2003 about 124 million cows produced nearly 500 million tons of raw milk. Around 350 to 500 million raw milk samples are analysed and 100 billion Euro are paied for cows milkall over the world (Baumgartner and Landgraf, 2005). Therefore the routine raw milk analysis plays a basic role in all milk payment and DHI (Dairy Herd Improvement) schemes world wide. Precise and right data are the key factor for fair payment of raw milk and expressive and correct milk production data as well as breeding values and selection data. The routine methods used are powerful tools to gain mass data in short time and with reasonably low costs. But routine methods have to be calibrated carefully and steadily to ensure, that the obtained data are „true”. The truth is defined with so called reference methods, which give a reference value for a defined sample (Baumgartner and Landgraf, 2005).

Also the checking of time measurement stability of the instruments during inter-calibration interval is very important activity. In the last time the row of authors was interested in calibration procedures of various methods of infraanalyse on various milk component measurements, fluoro-opto-electronic method on comatic cell count determination in milk and stability of results and samples in time at indirect methods of milk analyses (Sjaunja, 1984 a, b, c; Sjaunja et al., 1984; Sjaunja and Andersson, 1985; Hanuš et al., 1992 c, 1993 a, b, c, 1995, 1997, 2001, 2002, 2003, 2008; Tsenkova et al., 2000; Kukačková et al., 2000; Jankovská and Šustová, 2003; Kráčmar et al., 2004; Šustová et al., 2006, 2007; Hering et al., 2008). According to ČSN 590536 the instruments have to be calibrated quarterly. Of course, the more frequent calibration on results of reference methods is better for analytical result reliability. The routine analytical results for above mentioned purposes are obtained in laboratories of CMBA in the Czech Republic. The calibration is carried out monthly it means eleven times per year in this laboratory system. It is also important because of excluding of pos sible seasonal effects in composition of measured components on agreement between reference and indirect methods, in particular in fat and crude protein. In spite of the interval between consecutive calibrations is relatively short in this scheme sometimes it can be too long for quality keeping of time stability control samples, so called pilots. Various materials and ways of pilot sample preparation were investigated. From viewpoint of milk sample stability (preservation, cooling, freezing), pilot sample preparation and consequent checking of analytical instrument time stability (indirect methods) for measurement of main milk components, somatic cell counts and total mesophilic bacteria counts the row of studies was processed (Ng-Kwai-Hang and Hayes, 1982; Biggs et al., 1984; Szijarto and Barnum, 1984; Lintner et al., 1984; Kroger, 1985; Vines et al., 1986; Voort et al., 1987; Sato et al., 1985, 1987; Coleman and Moss, 1989; Szijarto et al., 1990; Valenberg, 1990; Hill et al., 1991; Hanuš et al., 1992 a, b, d; Genčurová et al., 1993; Benda, 1995; Tomáška et al., 2006). Only information to question of deep freezing for pilot sample stability are missing or are sporadic respectively (Hanuš et al., 2002). Therefore it was approached to treatment of standard milk pilot samples by immediate deep freezing by submersion of sample vials into liquid nitrogen $\left(\mathrm{N}_{2}\right)$ because of assurance of their long-term quality. This treatment way went out from presupposition that by quick freezing all biological processes in raw milk will be stoped. It should assure the microbiological quality of pilot samples. Further it should not come about a larger macromolecular and cellular (somatic cells and fat globules) destruction of milk components. The quick deep freezing should not enable the creation of larger crystals in milk sample.

Hypothetically, if deep freezing does not damage the macromolecular organic and cellular milk structures the pilot samples for checking of measurement stability of automatic instruments which work on indirect method principle could be used for considerably long periods without a charge change. Therefore the aim of this work was to investigate and verify the stability of long-term stored and deep frozen pilot samples for purposes of usage to checking of time stability of dairy analytical instrument measurement. Enhancement of pilot sample stability and longevity could improve the reliability of milk analytical results and consequently also the quality and safety of milk food chain.

\section{MATERIAL AND METHODS}

Raw cow milk from herds of School agricultural enterprise of Czech Agricultural University in Lány was used for creation of pilot standard samples (reference samples). Milk was obtained from Požáry herd, where the dairy cows of Jersey (J) breed are kept (57 closed lactations in control year 2006-2007) and where it is possible to expect the higher contents of milk components and from Ruda herd, where 337 lactations of Holstein $(\mathrm{H})$ breed dairy cows were closed in total with presupposition of lower milk component contents for assurance of maximal natural range of milk matter composition. Cooled tank milk was sampled of PACKO equipment and further was transported in cooling box to CMBA laboratory in Buštěhrad.

Milk was distributed into vials during continuous mixing in the laboratory. The vials have $50 \mathrm{ml}$ volume with screw cap. Necessary tightness was assured in this way. $40 \mathrm{ml}$ of milk was put into each vial. These prepared pilot milk samples were transported to CMBA residence in Hradištko pod Mednikem where samples were stored in liquid nitrogen $\left(\mathrm{N}_{2}\right)$ bath with temperature $-196{ }^{\circ} \mathrm{C}$. Samples were frozen in a few moments and stored in nitrogen bath. Consequently, the pilot milk samples were in small series step by step transported in liquid nitrogen 
bath to laboratory in Buštěhrad. There were stored in freezing box at temperature $-21^{\circ} \mathrm{C}$ until at measurement on analytical instrument.

The values of pilot samples were measured by one apparatus with use measure principles of wholespectral infrared analyse with Michelson's interferometer and mathematical mechanism of Fourier's transformations for milk components and flow cytometry for somatic cell count (SCC) CombiFoss 6000 (Foss Electric, Denmark). The instrument was under regular calibrations, proficiency testing and their supervision from accredited (No. 1340, EN ISO 17025, accreditation certificate No. 124/2004) National Reference Laboratory for Raw Milk in Rapotín with good results. Apparatus was regularly calibrated according to the reference method results (standard ČSN 57 0536, by Röse-Gottlieb's method for fat content, by Kjeldahl's method for crude protein and casein contents, by polarimetric and gravimetric methods for lactose and SNF contents according to standard ČSN 57 0530, by ureolytical conductometric method (Ureakvant; Hanuš et al., 1995, 1997, 2001, 2008; Hering et al., 2008) for milk urea concentration and by direct microscopy method according to ČSN EN ISO 13366-3 for SCC). The estimated extensive combined result uncertainties (1.96 times the combined uncertainty as standard deviation with probability level $95 \%$ ) for main milk components were: $\pm 2.77 \%$ for fat ( $\pm 0.101 \%$ for original unit), $\pm 2.59 \%$ for protein $( \pm 0.085 \%)$ and $\pm 2.77 \%$ for lactose $( \pm 0.115 \%)$. The estimated extensive combined result uncertainty was $\pm 9.3 \%$ for $S C C \leq 900$ thousands $/ \mathrm{ml}$.

The first serie of samples (A; Tab. I) was focused on period of one inter-calibration interval (1 month). The whole scale of indicators was measured in pilot samples: fat F (\%); crude protein CP (\%); lactose monohydrate L (\%); casein C (\%); solid non fat SNF (\%); urea $\mathrm{U}(\mathrm{mg} / 100 \mathrm{ml})$; somatic cell count SCC (thousands/ml).

The second serie of frozen standards (B) was aimed at long-term stability during several months (from May to January, it means nine months; Tab. II). The several instrumental calibrations with certain small shifts were carried out in the course of analyses. These calibration drifts were compensated in consideration of measured values.

The results were evaluated by variability appreciation in time rows and by comparison of this variability with values of extensive combined result uncertainties of relevant method measurements. The uncertainties were estimated in the framework of validation minutes of accredited laboratory according to official procedures. The standard declared variability values (standard deviations; ČSN 57 0536; Biggs, 1967, 1972 a, b, 1978, 1979 a, b; Biggs et al, 1987; Grappin, 1987) for reproducibility and agreement of instrument results with reference values (reproducibilty, accuracy, up to \pm 0.02 and $\pm 0.06 \%$ ) were the further basis for comparison in general.

It is necessary to stress that quick sample de-freezing at large temperature gradient has not to be quite safe procedure according to practical methodical ex- perience. The vials can act explosively, crack and be destroyed. Therefore it is necessary to use the firm high-quality plastic vials with screw cap and to defrost them as far as after successive temperature relaxation from -196 to $-21^{\circ} \mathrm{C}$.

\section{RESULTS AND DISCUSSION}

\section{Short-term experiment}

Results from previous papers with similar design were used at evaluation of applied procedures (Biggs, 1972, 1978; Grappin, 1987; Hanuš et al., 1992 a, b, c, d, 1993 a, b; Genčurová et al., 1993).

In the short-term (A) experiment (Tab. I) this was not disrupted by new calibration with sure shift of adjusted measurement levels. The repeatability of pilot sample values of watched milk indicators is determined only by pilot sample and instrument properties (such as repeatability, reliability of zero point adjustment, measurement cuvette flushing efficiency and so on) in this case. The more important values (the reference for sure instrument and period) are in second table line (Tab. I), where the deep frozen samples were measured at first. It is possible to observe the effect of deep freezing of samples on individual indicators in the direction to no frozen samples (first line, Tab. I). The stability in time row in consideration of reference values (repeatability) is observable in the down direction (Tab. I). The same evaluation is valid in the graphs (Fig. 1). The impact of freezing on pilot milk samples was relatively small. In principle it was in the framework of acceptable value of measurement repeatability ( $\pm 0.02 \%)$ for compositional indicators. This effect was slightly higher for fat of J breed sample and higher was for SCC of $\mathrm{H}$ breed sample. That is first important positive findings. Also the results of previous paper with slower frozen milk samples (normal freezing box, $-18{ }^{\circ} \mathrm{C}$ ) did not confirm a significant change in SCC determination. (Hanuš et al., 2002). The stability of values of frozen pilot samples (FPSs) was better according to standard deviations for important $\mathrm{F}$ evaluation in $\mathrm{H}$ breed samples. The repeatability of values of FPSs in the time was very good for other compositional indicators (from $\pm 0.011 \%$ for SNF to $\pm 0.042 \%$ for C; from 0.655 to $0.788 \mathrm{mg} / 100 \mathrm{ml}$ for $\mathrm{U}$ ). That is second important positive findings. The repeatability was better for SCC in J breed samples ( \pm 16.5 thousands $/ \mathrm{ml}$ ). Therefore it is possible to assess the higher variability it means worse repeatability $( \pm 30.1$ thousands $/ \mathrm{ml}$ ) in $\mathrm{H}$ breed sample less sceptically. The average values of milk indicators of FPSs are relatively close to values of original samples of both breeds for whole period generally. It is clear that the apparent result trends were not observable in the repeatability development of important milk indicators in FPSs at graphical evaluation (Fig. 1). The mild trend was only in CP. However, this trend covered absolutely very small shift (variation range). Mostly there are only oscillations in the result development. However, at first sight it is apparent between samples of both breeds 


\begin{tabular}{|c|c|c|c|c|c|c|c|c|c|c|c|c|c|c|}
\hline & $u$ & $\begin{array}{l}-\vec{b} \\
i \\
i\end{array}$ & $\begin{array}{l}8 \\
\vdots \\
0\end{array}$ & $\begin{array}{l}8 \\
\dot{i} \\
i\end{array}$ & $\begin{array}{l} \pm \\
\vdots \\
0 \\
\dot{0}\end{array}$ & $\begin{array}{l}\vec{b} \\
\dot{i}\end{array}$ & $\begin{array}{l}\bullet \\
\vdots \\
:\end{array}$ & 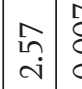 & \begin{tabular}{c|c}
$\bar{\delta}$ & \\
8 & 0 \\
0 & 0
\end{tabular} & \begin{tabular}{l|l}
-1 & 0 \\
$\vdots$ & 0 \\
$j$ & 0 \\
0
\end{tabular} & $\begin{array}{l}- \\
i \\
i\end{array}$ & $\mid \begin{array}{c}1 \\
\vdots \\
0 \\
0\end{array}$ & 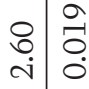 & \\
\hline & U & 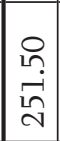 & 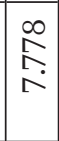 & $\begin{array}{c}8 \\
\stackrel{1}{2} \\
\hat{2} \\
-1\end{array}$ & 斊 & $\begin{array}{l}\hat{0} \\
\dot{0} \\
\dot{\nu}\end{array}$ & $\begin{array}{l}0 \\
\overrightarrow{6}\end{array}$ & 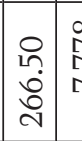 & 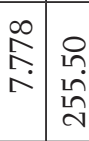 & 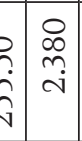 & $\begin{array}{l}\hat{0} \\
\dot{m} \\
\tilde{N}\end{array}$ & $\begin{array}{l}\stackrel{0}{\mathcal{N}} \\
\stackrel{\sim}{\sim} \\
\dot{\sim}\end{array}$ & 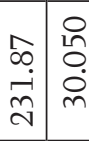 & \\
\hline & $\triangleright$ & & & 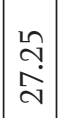 & $\mid \begin{array}{c}\overrightarrow{8} \\
\stackrel{0}{-i}\end{array}$ & $\begin{array}{l}\stackrel{8}{0} \\
\stackrel{0}{\circ}\end{array}$ & 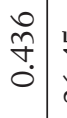 & 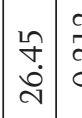 & 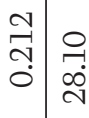 & 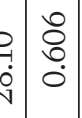 & $\begin{array}{l}\hat{N} \\
\grave{\lambda} \\
\text { त. }\end{array}$ & $\begin{array}{l}\tilde{o} \\
0 \\
0 \\
0\end{array}$ & 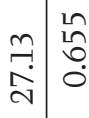 & \\
\hline$\stackrel{5}{\frac{0}{0}}$ & 㟶 & $\begin{array}{l}\stackrel{N}{\widehat{C}} \\
\infty\end{array}$ & $\begin{array}{l}T \\
0 \\
0 \\
0\end{array}$ & $\begin{array}{l}0 \\
0 \\
\infty \\
\infty\end{array}$ & $\mid \begin{array}{l}n \\
\tilde{o} \\
0 \\
0\end{array}$ & $\begin{array}{l}\stackrel{2}{\curvearrowright} \\
\infty\end{array}$ & $\begin{array}{l}\tilde{a} \\
\vdots \\
0 \\
0\end{array}$ & \begin{tabular}{|l|l}
$\infty$ & $n$ \\
$\infty$ & \\
$\infty$ &
\end{tabular} & \begin{tabular}{c|c|c}
1 & $\infty$ \\
\hdashline & 0 \\
0 & 0
\end{tabular} & \begin{tabular}{ll|l}
0 \\
0 \\
0 \\
0 & 0 \\
0 & 0 \\
0
\end{tabular} & $\mid \begin{array}{c}0 \\
0 \\
\infty \\
\infty\end{array}$ & $\mid \begin{array}{c}0 \\
\tilde{\tilde{o}} \\
0 \\
0\end{array}$ & 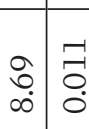 & \\
\hline & -1 & $\mid \begin{array}{l}\hat{\gamma} \\
\dot{\gamma}\end{array}$ & $\begin{array}{l}8 \\
8 \\
0\end{array}$ & $\begin{array}{c}\vec{\sigma} \\
\dot{\forall}\end{array}$ & $\mid \begin{array}{l}-\vec{a} \\
0 \\
\dot{0}\end{array}$ & $\begin{array}{l}\tilde{r} \\
\stackrel{\forall}{+}\end{array}$ & $\begin{array}{l}7 \\
\vdots \\
0 \\
0\end{array}$ & 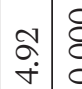 & 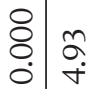 & 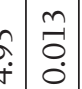 & $\begin{array}{l}n \\
\dot{\gamma} \\
\dot{f}\end{array}$ & $\left|\begin{array}{l|}0 \\
\multirow{1}{1}{} \\
0 \\
\dot{0}\end{array}\right|$ & 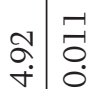 & \\
\hline & 0 & 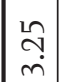 & $\begin{array}{l}8 \\
8 \\
0\end{array}$ & $\begin{array}{l}\tilde{m} \\
\tilde{m}\end{array}$ & $\left|\begin{array}{c}0 \\
0 \\
0 \\
0\end{array}\right|$ & $\begin{array}{l}\text { त̦ } \\
\text { m. }\end{array}$ & $\begin{array}{l}\vdots \\
\vdots \\
\vdots\end{array}$ & 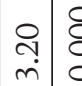 & 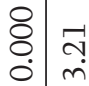 & \begin{tabular}{l|l|l}
4 & $n$ \\
& 0 \\
0 & 0 \\
0
\end{tabular} & $\mid \begin{array}{c}\circ \\
\text { ma } \\
\text { | }\end{array}$ & 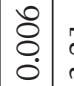 & \begin{tabular}{c|c}
$\vec{c}$ & \multicolumn{1}{c}{} \\
& 0 \\
0
\end{tabular} & \\
\hline & 山 & \begin{tabular}{|l|}
$\stackrel{2}{0}$ \\
$\stackrel{+}{+}$
\end{tabular} & $\begin{array}{l}0 \\
\tilde{n} \\
0 \\
0\end{array}$ & $\mid \begin{array}{l}\hat{0} \\
\dot{\forall}\end{array}$ & $\mid \begin{array}{l}0 \\
8 \\
0 \\
0\end{array}$ & $\begin{array}{l}\stackrel{8}{ } \\
\dot{\forall}\end{array}$ & $\begin{array}{l}8 \\
\vdots \\
\vdots\end{array}$ & 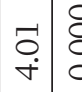 & 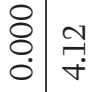 & 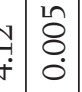 & 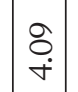 & $\left|\begin{array}{c}0 \\
0 \\
0 \\
0 \\
0\end{array}\right|$ & 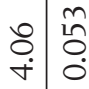 & \\
\hline & 0 & \begin{tabular}{|l|}
$\infty$ \\
$\sim$ \\
$n$ \\
$n$
\end{tabular} & $\begin{array}{l}2 \\
0 \\
0 \\
0 \\
0\end{array}$ & $\stackrel{\hat{A}}{\mathrm{~m}}$ & 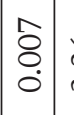 & $\begin{array}{l}\stackrel{0}{ } \\
m \\
m\end{array}$ & $\begin{array}{l}0 \\
\stackrel{0}{0} \\
\vdots \\
0\end{array}$ & \begin{tabular}{l|l}
$\infty$ & \\
$\stackrel{\infty}{m}$ & \\
$m$
\end{tabular} & 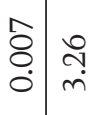 & 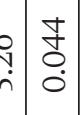 & $\mid \begin{array}{c}\infty \\
\sim \\
m \\
m\end{array}$ & 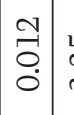 & \begin{tabular}{l|l}
$\stackrel{n}{c}$ & $\stackrel{f}{0}$ \\
$m$ & 0
\end{tabular} & \\
\hline & $\begin{array}{l}0 \\
\text { U }\end{array}$ & 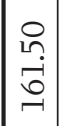 & $\begin{array}{l}\alpha \\
\sigma \\
\sigma \\
\sigma\end{array}$ & $\begin{array}{l}\stackrel{0}{1} \\
\stackrel{1}{\sim} \\
\stackrel{\sim}{-}\end{array}$ & 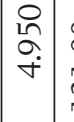 & $\begin{array}{l}8 \\
\dot{m} \\
\vec{y}\end{array}$ & 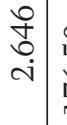 & 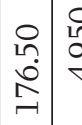 & 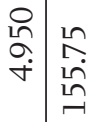 & 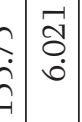 & $\mid \begin{array}{c}\hat{0} \\
\hat{1} \\
0 \\
\end{array}$ & 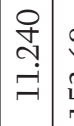 & \begin{tabular}{l|l}
$\stackrel{+}{0}$ & $\stackrel{n}{0}$ \\
\\
$\tilde{n}$ & 0 \\
& -
\end{tabular} & \\
\hline & $\triangleright$ & & & $\begin{array}{l}8 \\
\vec{i}\end{array}$ & $\begin{array}{l}\hat{0} \\
\hat{0}\end{array}$ & $\begin{array}{l}\hat{\sigma} \\
\dot{d} \\
\hat{v}\end{array}$ & 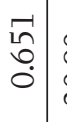 & 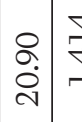 & 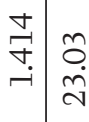 & 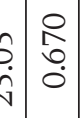 & $\left|\begin{array}{l}\stackrel{0}{ } \\
\tilde{M} \\
\vec{i}\end{array}\right|$ & 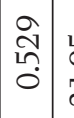 & \begin{tabular}{l|l} 
& $\infty$ \\
& $\infty$ \\
& $\stackrel{0}{0}$
\end{tabular} & \\
\hline & 㞫 & $\stackrel{8}{\circ}$ & $\begin{array}{l}n \\
0 \\
0 \\
0\end{array}$ & ণั. & $\mid \begin{array}{l}\overrightarrow{1} \\
\overrightarrow{0} \\
\dot{0}\end{array}$ & $\begin{array}{l}\stackrel{q}{a} \\
\text { a. }\end{array}$ & 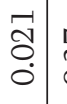 & $\begin{array}{l}\hat{\sigma} \\
\sigma\end{array}$ & 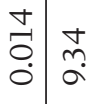 & $\begin{array}{lll}+ & \hat{0} \\
2 & 0 \\
0 & 0\end{array}$ & aे & 旁 & \begin{tabular}{c|c|c}
$\infty$ & $\tilde{o}$ \\
$\curvearrowright$ & $\tilde{O}$
\end{tabular} & \\
\hline & الـ & \begin{tabular}{|l|}
$\stackrel{+}{\infty}$ \\
$\stackrel{+}{+}$
\end{tabular} & $\begin{array}{l}\hat{0} \\
0 \\
0 \\
0\end{array}$ & $\mid \begin{array}{c}\infty \\
\infty \\
\dot{\sigma}\end{array}$ & $\begin{array}{l}0 \\
8 \\
0 \\
0\end{array}$ & $\begin{array}{l}\tilde{0} \\
\dot{\forall} \\
\dot{\forall}\end{array}$ & $\begin{array}{l}0 \\
\vdots \\
\vdots \\
0\end{array}$ & 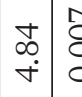 & \begin{tabular}{c|c}
5 & $\circ$ \\
$\vdots$ & $\infty$ \\
\hdashline & \multirow{+}{*}{}
\end{tabular} & \begin{tabular}{l|l|}
$\stackrel{f}{0}$ \\
$\stackrel{5}{0}$ \\
$\dot{f}$
\end{tabular} & \begin{tabular}{l|}
$\dot{ }$ \\
$\infty$ \\
$\dot{+}$ \\
\end{tabular} & $\begin{array}{l}\hat{a} \\
\stackrel{0}{0}\end{array}$ & 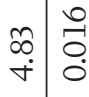 & \\
\hline & ن & $\mid \begin{array}{l}\qquad \\
\stackrel{n}{\circ} \\
\dot{r}\end{array}$ & $\begin{array}{l}0 \\
\vdots \\
0\end{array}$ & $\begin{array}{l}\dot{t} \\
\dot{+}\end{array}$ & $\mid \begin{array}{l}0 \\
8 \\
0 \\
0\end{array}$ & 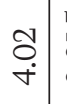 & $\begin{array}{l}2 \\
\vdots \\
\vdots \\
0\end{array}$ & $\underset{+}{\stackrel{c}{*}}$ & 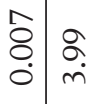 & $\begin{array}{l}n \\
\tilde{n} \\
0 \\
0\end{array}$ & $\underset{+}{\overrightarrow{+}}$ & $\begin{array}{l}n \\
\vdots \\
0 \\
0\end{array}$ & \begin{tabular}{l|l}
$\stackrel{1}{o}$ & $\stackrel{\partial}{\partial}$ \\
$\stackrel{+}{\circ}$
\end{tabular} & \\
\hline & H & 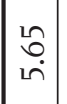 & $\begin{array}{l}n \\
0 \\
0 \\
0\end{array}$ & 尽 & 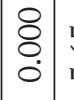 & $\begin{array}{l}\stackrel{L}{0} \\
\stackrel{\leftrightarrow}{n}\end{array}$ & 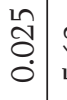 & $\begin{array}{l}\text { ç } \\
\text { ம். }\end{array}$ & 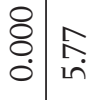 & $=\begin{array}{l}a \\
\dot{v} \\
0 \\
0 \\
0\end{array}$ & 站 & $\vec{I}$ & 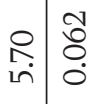 & \\
\hline & & $x$ & 宓 & $x$ & $\tau_{\infty}$ & $x$ & $\vec{m}$ & $x \bar{v}$ & 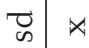 & $x=\vec{c}$ & $x$ & $\vec{\sigma}$ & $\times \bar{c}$ & \\
\hline & 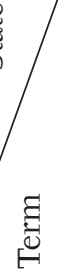 & 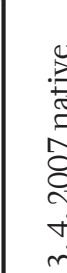 & ¿ & 0 & & $\begin{array}{l}\hat{\circ} \\
\dot{+} \\
\text { c่ }\end{array}$ & & $\begin{array}{l}\text { o } \\
\dot{+} \\
\dot{0}\end{array}$ & & $\begin{array}{l}\hat{0} \\
\dot{+} \\
\dot{m}\end{array}$ & ம் & & 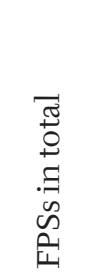 & \\
\hline
\end{tabular}



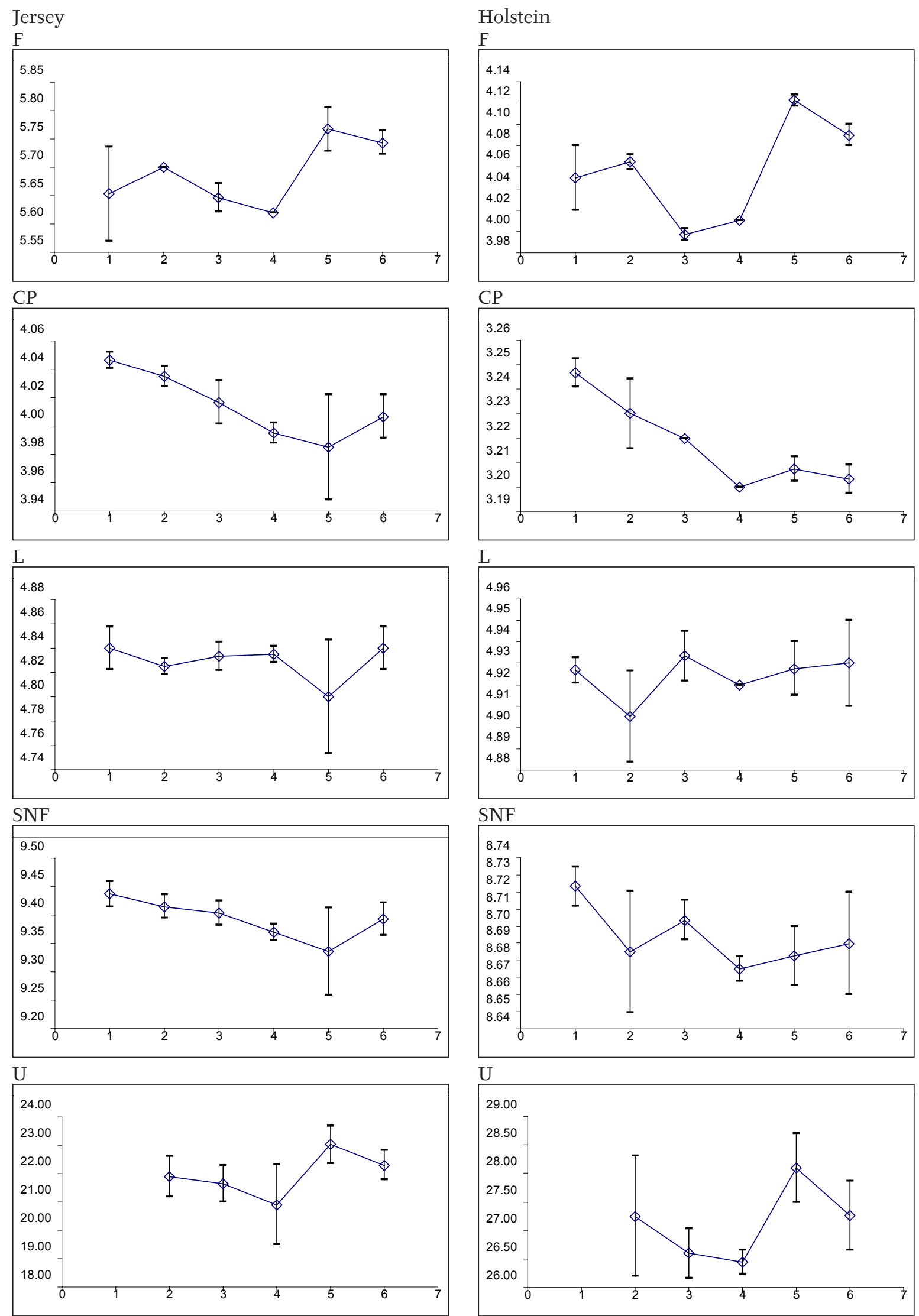

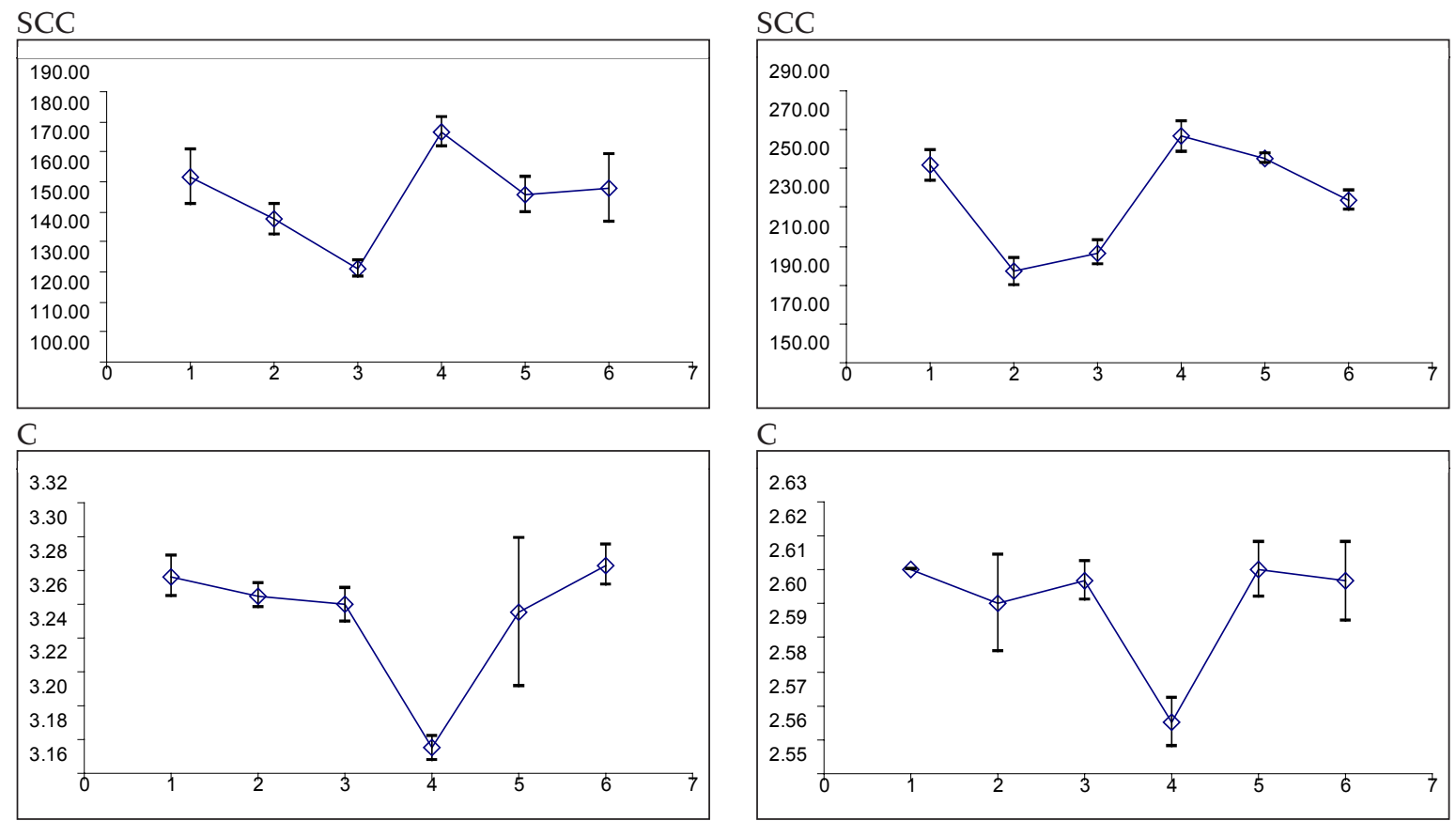

1: Graphical notation of value stability of FPS measurement in short-term experiment

( $\mathrm{x}$ axis = component concentration; $\mathrm{y}$ axis = time order of pilot sample measurement)

(Fig. 1) that oscillations in the course of curves are very markedly similar. Therefore it is possible to attribute these oscillations rather to instrument effects than to sample effects as freezing and storage technology. That is third important positive findings. The applied A procedure is useable for checking of instrumental measurement stability in routine laboratories for testing of important milk quality indicators in one month period.

\section{Long-term experiment}

In the long-term (B) experiment (Tab. II) the process of result repeatability was a little influenced by regular month calibrations of used instrument and also by one weightier reparation. The frozen sample reference values are on fourth line of Tab. II. The value oscilations were markedly in this experiment. So the results were worse. The protein values in J breed sample showed the worst repeatability results. The courses of other curves size up as acceptable. It is visible between two breed samples (Fig. 2) that oscilations are very similar in curve courses in fat and lactose. That is reason why it is possible to attribute them more to instrumental effects than to sample effects. The repeatability values (standard deviations) of FPSs were mostly lower than $\pm 0.06 \%$. It is good acceptable for result agreement of indirect method with reference method (ČSN 57 0536, $\pm 0.07 \%)$. Relevant standard deviations varied from $\pm 0.025 \%$ for L to \pm 0.059 \% for F (in J breed) with exception CP $\pm 0.085 \%$ in J breed sample. These were also mostly lower than values of extensive combined uncertainty of measurement results which are introduced in laboratories for indirect method of milk infraanalyse ( $\mathrm{F} \pm 0.101, \mathrm{CP} \pm 0.085$ and $\mathrm{L} \pm 0.115 \%$ ) in current range of variability of measured values. The results showed the ability of long-term stability of FPSs for basic milk indicators. Also B procedure was shown in spite of sure disturbance in curve courses as applicable for instrumental measurement stability control in routine laboratories for milk composition testing in half-year period of storage. However, in practical use one reference value for concrete FPS serves from calibration to next calibration. It means for one month and new reference value for repeatability control (instrument stability) is determined after each calibration in the case of milk components in the Czech Republic. So the persistent trend in measurement repeatability of FPSs can indicate an inadequate instrumental drift in the results.

Baumgartner and Landgraf (2005) mentioned the good result stability (fat and protein, protein stability was better) of specific raw milk deep frozen standards (special freezing procedure with storage at $-20^{\circ} \mathrm{C}$ ) with reference method values and with long shelf life as suitable way for creation of reference system with feed-back about comparison and control of result stability.

\section{CONCLUSION}

The development of technology and testing of pilot milk samples deep freezing gave the applicable results for practical use in routine testing laboratories for milk quality control in terms of time stability checking of instruments for determination of main milk components (fat, protein, lactose and urea) and somatic cell count. The reference value of sure milk indicator is determined always by concrete in- 
II: Long-term experiment results with repeatability of values of deep frozen milk pilot samples (FPSs) at infraanalyse measurement

\begin{tabular}{|c|c|c|c|c|c|c|c|c|c|c|c|c|c|}
\hline \multirow{3}{*}{ Term State } & & \multicolumn{6}{|c|}{ Jersey } & \multicolumn{6}{|c|}{ Holstein } \\
\hline & & \multicolumn{2}{|c|}{ F } & \multicolumn{2}{|c|}{$\mathrm{CP}$} & \multicolumn{2}{|c|}{ L } & \multicolumn{2}{|c|}{$\mathrm{F}$} & \multicolumn{2}{|c|}{$\mathrm{CP}$} & \multicolumn{2}{|c|}{$\mathrm{L}$} \\
\hline & & $\mathrm{x}$ & sd & $\mathrm{x}$ & sd & $\mathrm{x}$ & sd & $\mathrm{x}$ & sd & $\mathrm{x}$ & sd & $\mathrm{x}$ & sd \\
\hline $\mathrm{N}$ & 1 & 5.22 & 0.012 & 3.90 & 0.028 & 4.88 & 0.036 & 3.71 & 0.015 & 3.18 & 0.006 & 4.90 & 0.015 \\
\hline NP & 2 & 5.17 & 0.015 & 3.91 & 0.010 & 4.88 & 0.026 & 3.76 & 0.006 & 3.21 & 0.006 & 4.92 & 0.012 \\
\hline NP & 3 & 5.31 & 0.020 & 3.92 & 0.015 & 4.90 & 0.012 & 3.74 & 0.010 & 3.22 & 0.006 & 4.91 & 0.006 \\
\hline 1.6. 2007 & 4 & 5.24 & 0.008 & 3.98 & 0.005 & 4.87 & 0.010 & 3.78 & 0.000 & 3.26 & 0.005 & 4.90 & 0.010 \\
\hline 20.6 .07 & 5 & & & & & & & 3.77 & 0.013 & 3.24 & 0.010 & 4.88 & 0.022 \\
\hline 28.6 .07 & 6 & 5.14 & 0.026 & 3.90 & 0.006 & 4.82 & 0.006 & 3.77 & 0.000 & 3.24 & 0.000 & 4.90 & 0.012 \\
\hline 30.8 .07 & 7 & 5.18 & 0.015 & 3.85 & 0.008 & 4.87 & 0.010 & 3.83 & 0.010 & 3.36 & 0.006 & 4.87 & 0.005 \\
\hline 21.9 .07 & 8 & 5.16 & 0.006 & 3.77 & 0.006 & 4.85 & 0.012 & 3.82 & 0.006 & 3.33 & 0.012 & 4.85 & 0.015 \\
\hline 10. 10.07 & 9 & 5.16 & 0.006 & 3.73 & 0.012 & 4.92 & 0.012 & 3.80 & 0.006 & 3.30 & 0.006 & 4.92 & 0.012 \\
\hline 23.10 .07 & 10 & 5.13 & 0.005 & 3.74 & 0.005 & 4.92 & 0.015 & 3.79 & 0.005 & 3.31 & 0.006 & 4.92 & 0.010 \\
\hline 19. 11.07 & 11 & 5.29 & 0.005 & 3.71 & 0.005 & 4.85 & 0.014 & 3.92 & 0.006 & 3.29 & 0.010 & 4.86 & 0.014 \\
\hline 10.12 .07 & 12 & 5.25 & 0.008 & 3.70 & 0.012 & 4.87 & 0.013 & 3.89 & 0.006 & 3.29 & 0.006 & 4.86 & 0.013 \\
\hline 12.12 .07 & 13 & 5.10 & 0.012 & 3.72 & 0.000 & 4.86 & 0.006 & 3.80 & 0.000 & 3.29 & 0.006 & 4.86 & 0.006 \\
\hline 18. 12.07 & 14 & 5.13 & 0.010 & 3.75 & 0.005 & 4.87 & 0.010 & 3.79 & 0.006 & 3.32 & 0.008 & 4.86 & 0.013 \\
\hline 4. 1.2008 & 15 & 5.12 & 0.017 & 3.72 & 0.000 & 4.87 & 0.006 & 3.79 & 0.005 & 3.30 & 0.006 & 4.87 & 0.013 \\
\hline 11.1 .08 & 16 & 5.14 & 0.010 & 3.73 & 0.006 & 4.86 & 0.012 & 3.79 & 0.008 & 3.30 & 0.005 & 4.86 & 0.010 \\
\hline 17. 1.08 & 17 & 5.21 & 0.010 & 3.73 & 0.010 & 4.88 & 0.000 & 3.84 & 0.008 & 3.30 & 0.000 & 4.88 & 0.005 \\
\hline FPSs only (od 4) & $\mathrm{x}$ & 5.17 & & 3.77 & & 4.87 & & 3.81 & & 3.29 & & 4.88 & \\
\hline & $\mathrm{sd}$ & 0.059 & & 0.085 & & 0.027 & & 0.043 & & 0.033 & & 0.025 & \\
\hline
\end{tabular}

(N new native samples; NP native only preserved samples; FPSs start from order 4)

Jersey
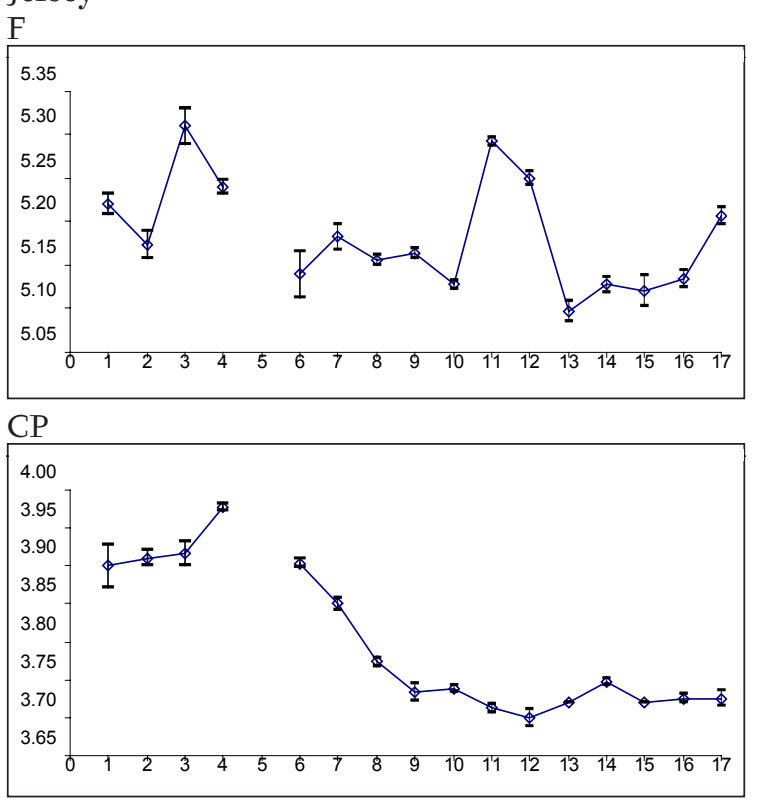

Holstein

F

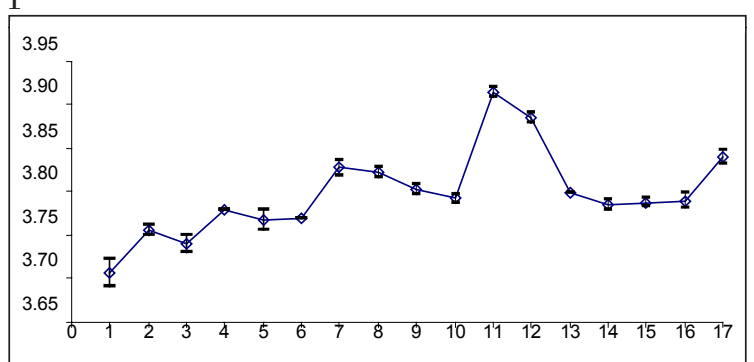

CP

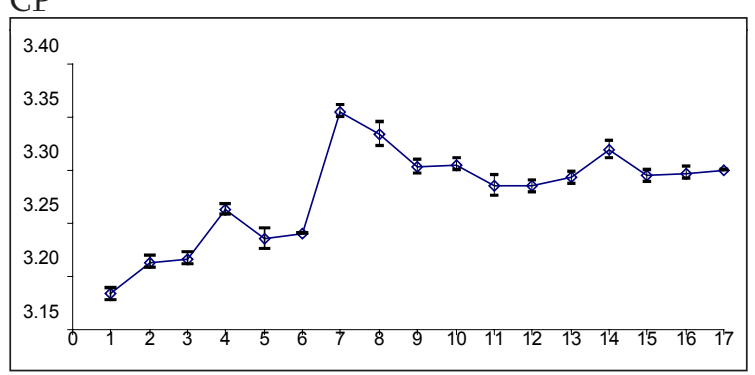



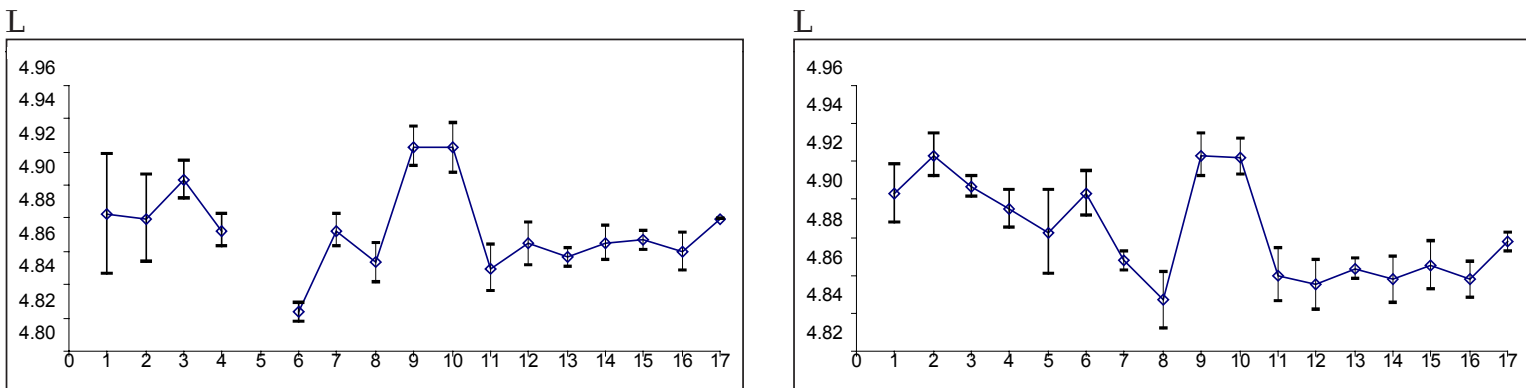

2: Graphical notation of value stability of FPS measurement in long-term experiment

( $\mathrm{x}$ axis $=$ component concentration; $\mathrm{y}$ axis = time order of pilot sample measurement)

strument and is valid for this instrument for relevant period. In the laboratories an important thing will be to determine the individual discrimination limits (for suitable results) for evaluation of instrumental measurement stability checking according to own statistical data by relevant procedure. The FPS method can improve the the result quality assurance in milk testing laboratories. It is possible to recommend application of one FPS with current mean milk composition for instrument measurement stability control without another sample with more different composition.

\section{SUMMARY}

Essential part of raw milk component measurement is indirect analyse on the basis of infrared spectrum measurement. Milk analyses are important because of needs of milk recording for cattle genetical improvement purposes and also because of milk quality determination for technological processing procedures. It is necessary to calibrate the instruments regularly. Also the checking of time measurement stability is important. The preparation of pilot samples for mentioned puropses was described. However, information about deep frozen pilot sample stability are sporadic. Deep freezing of pilot samples should assure their microbiological quality. It should not come about a larger macromolecular and cellular destruction of milk components. The quick deep freezing should not enable the creation of larger crystals in milk. Aim of this work was to investigate and verify the stability of long-term stored and deep frozen pilot samples for mentioned purposes. Enhancement of pilot sample stability and longevity could improve the reliability of milk analytical results and also quality and safety of milk food chain. Pilot samples were frozen in liquid nitrogen bath $-196^{\circ} \mathrm{C}$ and after that stored at $-21{ }^{\circ} \mathrm{C}$ for one month (A) and more months (B) till analyse. The pilot sample values were measured by CombiFoss 6000: fat F (\%); protein CP (\%); lactose L (\%); casein C (\%); solid non fat SNF (\%); urea U (mg/100 ml); somatic cell count SCC (ths./ml). Calibration drifts were compensated. Pilot sample result stability was evaluated by variability in time rows and also by comparison to result uncertainties. In the short-term (A) experiment the impact of freezing on pilot milk samples was relatively small. In principle it was in the framework of acceptable value of measurement repeatability $( \pm 0.02 \%)$ for compositional indicators. This effect was slightly higher for fat of J (Jersey) breed sample and higher was for SCC of H (Holstein) breed sample. The stability of values of frozen pilot samples (FPSs) was better according to standard deviations for important $\mathrm{F}$ evaluation in $\mathrm{H}$ breed samples. The repeatability of values of FPSs in the time was very good for other compositional indicators. The repeatability was better for SCC in J breed samples ( \pm 16.5 thousands $/ \mathrm{ml})$. Therefore it is possible to assess the higher variability it means worse repeatability ( \pm 30.1 thousands $/ \mathrm{ml}$ ) in $\mathrm{H}$ breed sample less sceptically. The apparent result trends were not observable in the repeatability development of important milk indicators in FPSs. The mild trend was only in CP. However, this trend covered absolutely very small shift (variation range). Mostly there are only oscillations in the result development, which are similar between $\mathrm{J}$ and $\mathrm{H}$ breed samples. Therefore it is possible to attribute these oscillations rather to instrument effects than to sample effects. The value oscilations were markedly higher in the longterm (B) experiment. So the results were worse. However, the courses of other curves size up as acceptable. It is visible between two breed samples that oscilations are very similar in curve courses in fat and lactose. That is reason why it is possible to attribute them more to instrumental effects than to sample effects. The repeatability values (standard deviations) of FPSs were mostly lower than $\pm 0.06 \%$. It is good acceptable for result agreement of indirect method with reference method. Relevant standard deviations varied from $\pm 0.025 \%$ for $\mathrm{L}$ to $\pm 0.059 \%$ for $\mathrm{F}$ (in J breed) with exception CP $\pm 0.085 \%$ in J breed sample. Also B procedure was shown in spite of sure disturbance in curve courses as applicable for instrumental measurement stability control in routine laboratories for milk composition testing 
in half-year period of storage. However, in practical use one reference value for concrete FPS serves from calibration to next calibration. So the persistent trend in measurement repeatability of FPSs can indicate an inadequate instrumental drift in the results. The applied FPS procedure is usable for stability control of instrumental measurement in routine laboratories for testing of important milk quality indicators.

\section{SOUHRN}

Validace hlubokého zamrazování pilotních vzorků pro kontrolu stability výsledků nepřímých analýz základního složení mléka a pro jejich dlouhodobou údržnost

Podstatnou částí měření složek syrového mléka je nepřímá analýza na základě měření infračerveného spektra. Analýzy mléka jsou důležité pro potřeby kontroly užitkovosti dojnic k účelům genetického zušlechtování skotu a také k určení kvality mléka pro technologicko-zpracovatelské postupy. Je nezbytné přístroje pravidelně kalibrovat. Také kontrola časové stability měření je důležitá. Příprava pilotních vzorků pro zmíněné účely byla popsána. Informace o stabilitě hluboce zmražených pilotních vzorků jsou však sporadické. Hluboké zmrazování pilotních vzorků by mělo zajistit jejich mikrobiologickou kvalitu. Nemělo by dojít k většímu makromolekulárnímu a buněčnému poškození mléčných složek. Rychlé hluboké zmražení by nemělo umožnit tvorbu větších krystalů v mléce. Cílem této práce bylo vyšetřit a ověřit stabilitu dlouhodobě uložených a hluboce zmražených pilotních vzorků pro naznačené účely. Zvýšení stability a dlouhověkosti pilotních vzorků by mohlo zlepšit věrohodnost mléčných analytických výsledkủ a také kvalitu a bezpečnost mléčného potravinového řetězce. Pilotní vzorky byly zmraženy v tekutém dusíku $-196{ }^{\circ} \mathrm{C}$ a poté uloženy při $-21{ }^{\circ} \mathrm{C}$ po jeden měsíc (A) a více měsíců (B) do analýzy. Hodnoty pilotních vzorků byly měřeny pomocí CombiFoss 6000: tuk F (\%); bílkoviny CP (\%); laktóza L (\%); kasein C (\%); sušina tukuprostá SNF (\%); močovina U (mg/100 ml); počet somatických buněk SCC (tis./ml). Byly kompenzovány kalibrační posuny. Výsledky stability pilotních vzorků byly hodnoceny pomocí variability v časových řadách a také pomocí srovnání k výsledkovým nejistotám. V krátkodobém pokusu (A) byl vliv zamražení pilotních vzorků mléka poměrně malý, v podstatě v rámci hodnoty povolené opakovatelnosti ( $\pm 0,02 \%)$ pro složkové ukazatele. Mírně byl vyšší u tuku u vzorku plemene Jersey (J) a vyšší byl u SCC u vzorků plemene Holštýn (H). Stabilita hodnot zamražených pilotních vzorků (FPSs) podle směrodatných odchylek pro důležité hodnocení F byla lepší pro vzorky plemene H. U ostatních složkových ukazatelů byla opakovatelnost hodnot FPSs velmi dobrá. U SCC byla opakovatelnost lepší u FPSs plemene J ( $\pm 16,5$ tis./ml). Větší variabilitu ( $\pm 30,1$ tis./ml) u vzorku H tak lze hodnotit méně skepticky. Nebyly pozorovatelné zjevné trendy výsledků ve vývoji opakovatelnosti v FPSs. Mírný trend je pouze u CP, kde pokrývá velmi malý obor kolísání. Většinou jsou ve vývoji výsledků oscilace. Tyto jsou podobné mezi vzorky J a H. Proto je lze přičítat přístrojovým, nikoliv vzorkovým vlivům. V dlouhodobém (B) pokusu byly oscilace hodnot zřetelně větší. Výsledky byly tedy horší. Průběhy většiny složkových křivek se však jeví jako přijatelné. Mezi dvěma vzorky plemen je patrné, že oscilace v průbězích křivek jsou si u tuku a laktózy velmi podobné. Proto je lze rovněž přičítat více přístrojovým vlivům než vzorkovým. Hodnoty opakovatelnosti (směrodatné odchylky) FPSs byly převážně nižší než $\pm 0,06$ \%, což je dobře přijatelné pro shodu výsledků nepřímé metody s referenční metodou. Pohybovaly se od $\pm 0,025 \%$ pro L do $\pm 0,059$ \% pro F (u plemene J), s výjimkou CP $\pm 0,085 \%$ u vzorku plemene J. I postup B se ukázal, přes určité disturbance v průběhu křivek, jako použitelný pro kontrolu stability měření přístrojů v rutinních laboratořích pro testování složení mléka v půlroční periodě uložení. V praktickém použití však slouží jedna referenční hodnota pro daný FPS od kalibrace ke kalibraci. Trvalý trend v opakovatelnosti měření FPSs tak může indikovat neadekvátní přístrojový drift ve výsledcích. Použitý postup FPS je použitelný pro kontrolu stability měření přístrojů v rutinních laboratořích pro testování důležitých ukazatelů kvality mléka.

mléko, pilotní vzorek, hluboké zmražení, dlouhodobá údržnost, kontrola stability měření, nepřímá metoda, infraanalýza, tuk, bílkoviny, počet somatických buněk

This work was supported by the research projects MSMT, MSM 2678846201 and INGO, LA 244 and was provided in framework of research activities of NRL-RM in Rapotín.

\section{REFERENCES}

BAUMGARTNER, CH., LANDGRAF, A., 2004: Deep frozen raw milk standards - The way from reference methods to reference system. 34th ICAR Session, May 28-June 3, Sousse, Tunisia, EAAP Publication 113, 2005: 253-257.
BENDA, P., 1995: The effect of some preservatives on natural microflora in milk samples. (In Czech) Veterinary Medicine - Czech, 40, 11, 359-364.

BIGGS, D. A., 1967: Milk analysis with the infrared milk analyzer. Journal of Dairy Science, 50, 799-803. 
BIGGS, D. A., 1972 a: Infrared milk analyzer. Journal of Dairy Science, 55, 5, 650-651.

BIGGS, D. A., 1972 b: Precision and accuracy of infrared milk analysis. Journal of Association Official Analytical Chemistry, 55, 3, 488-497.

BIGGS, D. A., 1978: Instrumental infrared estimation of fat, protein, and lactose in milk: collaborative study. Journal of Association Official Analytical Chemistry, 61, 5, 1015-1034.

BIGGS, D. A., 1979 a: Performance specifications for infrared milk analysis. Journal of Association Official Analytical Chemistry, 62, 6, 1211-1214.

BIGGS, D. A., 1979 b: Infrared estimation of fat, protein, and lactose in milk: Evaluation of Multispec instrument. Journal of Association Official Analytical Chemistry, 62, 6, 1202-1207.

BIGGS, D. A., JOHNSSON, G., SJAUNJA, L. O., 1987: Analysis of fat, protein, lactose, total solids by infra-red absorption. Bulletin of International Dairy Federation, Doc. 208, 21-29.

BIGGS, D. A., SZIJARTO, L.F., VOORT VAN DE, F. R., 1984: Fresh milk sampling for centralized milk testing. Journal of Dairy Science, 67: 3085-3092.

COLEMAN, D. A., MOSS, B. R., 1989: Effects of several factors on quantification of fat, protein, and somatic cells in milk. Journal of Dairy Science, 72, 3295-3303.

ČSN 57 0530: Methods for testing of milk and milk products. (In Czech) 1972: ČNI Praha.

ČSN 57 0536: Determination of milk composition by mid-infrared analyzer. (In Czech) 1999: ČNI Praha.

ČSN EN ISO 13366-3: Milk - Enumeration of somatic cells - Part 3: Fluoro-opto-electronic method. (In Czech) 1998: ČNI Praha.

GENČUROVÁ, V., HANUŠ, O., KOPECKÝ, J., JEDELSKÁ, R., 1993: Effect of milk sample treatment before measurement for reading of somatic cell number by the Fossomatic apparatus. (In Czech) Živočišná Výroba / Czech Journal of Animal Science, 38, 6, 555-565.

GRAPPIN, R., 1987: Definition and evaluation of the overall accuracy of indirect methods of milk analysis - aplication to calibration procedure and quality control in dairy laboratory. Bulletin of International Dairy Federation, Doc. 208, IDF Provisional Standard 128, 3-12.

HANUŠ, O., BENDA, P., GENČUROVÁ, V., 1992 a: Tests of Milkofix a new preservative substance for milk samples used for the purposes of an infrarad analysis of basic milk composition. Part I. Checks of bacteriostatic and bactericidal abilities and interferential effect. (In Czech) Veterinární Medicína Praha, 37, 1, 21-31.

HANUŠ, O., FICNAR, J., KOPECKÝ, J., JEDELSKÁ, R., BERANOVÁ, A., HAVLÍČKOVÁ, K., 1997: A retrospective study of results and evolution of methodical design for preparation of urea milk calibration standard sets. (In Czech) Výzkum v chovu skotu, 2, 7-21.

HANUŠ, O., GENČUROVÁ, V., ŽVÁČKOVÁ, I., 1992 b: Tests of Milkofix a new preservative sub- stance for milk samples used for the purposes of an infrarad analysis of basic milk composition. Part II.Checks of preservative effects in relation to the infrared analysis. (In Czech) Veterinární Medicína Praha, 37, 1, 33-43.

HANUŠ, O., GENČUROVÁ, V., GABRIEL, B., 1992 c: The effect of sample aging on the accuracy of an infrared analysis of basic milk composition. (In Czech) Veterinary Medicine - Czech, 37, 3, 149-160.

HANUŠ, O., GENČUROVÁ, V., KOPECKÝ, J., GABRIEL, B., 1993 a: The effect of a change in the discriminator level of fluorooptoelectronic determination of somatic cell counts on the results in raw milk and pasteurized milk samples. (In Czech) Živočišná Výroba / Czech Journal of Animal Science, 38, 5, 443-455.

HANUŠ, O., GENČUROVÁ, V., GABRIEL, B., KOPECKÝ, J., JEDELSKÁ, R., 1993 b: Calibration and accuracy control in determining somatic cell count in cows' milk by means of the method Fossomatic. (In Czech) Živočišná Výroba / Czech Journal of Animal Science, 38, 10, 907-926.

HANUŠ, O., GENČUROVÁ, V., GABRIEL, B., ŽVÁČKOVÁ, I., 1992 d: A comparison of the efficiency of Milkofix preservative substance with traditional preservatives used to determine somatic cell counts in milk samples by a fluoro-opto-electronic method. (In Czech) Veterinární Medicína Praha, 37, 2, 91-99.

HANUŠ, O., HERING, P., MOTYČKA, Z., JEDELSKÁ, R., 2002: Effect of milk samples freezing on practical interpretationability of individual somatic cell counts. (In Czech) Výzkum v chovu skotu, 2, 9-13.

HANUŠ, O., HERING, P., FRELICH, J., JÍLEK, M., GENČUROVÁ, V., JEDELSKÁ, R., 2008: Reliability of milk urea analyse results by various methods in use of artificial milk control samples. Czech Journal of Animal Science, 53, 4, 156-165.

HANUŠ, O., JÍLEK, M., FICNAR, J., BERANOVÁ, A., JEDELSKÁ, R., HAVLÍČKOVÁ, K., MÍČOVÁ, Z., 1995: Ways of preparing standards for calibration of indirect methods of determination of urea concentration in milk. (In Czech) Živočišná Výroba / Czech Journal of Animal Science, 40, 10, 441-451.

HANUŠ, O., KLIMEŠ, M., MIHULA, P., KOZÁKOVÁ, A., JEDELSKÁ, R., 2003: Impacts of the sampling of milk and the basic milk treatment on its freezing point and other compositional parameters. (In Czech) Výzkum v chovu skotu, 4, 10-17.

HANUŠ, O., PONÍŽIL, A., KOPECKYY, J., GABRIEL, B., JEDELSKÁ, R., 1993 c: Dynamics of variations of calibration line setups at determination of somatic cell counts of Fossomatic apparatus with various discriminator levels. (In Czech) Veterinary Medicine - Czech, 38, 8, 497-509.

HANUŠ, O., SKYVA, J., HOFBAUER, J., KLOPČIČ, M., GENČUROVÁ, V., JEDELSKÁ, R., 2001: Reliability of analytical methods applicable at milk urea determination. (In Czech) Acta universitatis 
agriculturae et silviculturae Mendelianae Brunensis (Brno), XLIX, 3, 143-154.

HERING, P., HANUŠ, O., FRELICH, J., PYTLOUN, J., MACEK, A., JANÜ, L., KOPECKY,, J., 2008: Relationships between the results of various methods of urea analysis in native and enriched milk. Czech Journal of Animal Science, 53, 2, 64-76.

HILL, A. R., MAKARCHUK, M. J., SZIJARTO, L. F., 1991: Comparison of alternative calibration procedures for infra-red milk analyzers. Canadian Institute Scentific Technology Journal, 24, 5, 228-232.

JANKOVSKÁ, R., ŠUSTOVÁ, K., 2003: Analysis of cow milk by near-infrared spectroscopy. Czech Journal of Food Science, 21, 4, 123-128.

KRÁČMAR, S., JANKOVSKÁ, R., ŠUSTOVÁ, K., KUCHTÍK, J., ZEMAN, J., 2004: Analysis of amino acid composition of sheep colostrum by nearinfrared spectroscopy. Czech Journal of Animal Science, 49, 5, 177-182.

KROGER, M., 1985: Milk sample preservation. Journal of Dairy Science, 68, 783-787.

KUKAČKOVÁ, O., ČURDA, L., JINDŘICH, J., 2000: Multivariate calibration of raw cow milk using NIR spectroscopy. Czech Journal of Food Science, 18, $1,1-4$.

LINTNER, T. J., HEALD, C. W., EBERHART, R. J., 1984: Somatic cell reference samples for calibration of milk somatic cell counting methods. Journal of Food Protection, 47, 694-696.

NG-KWAI-HANG, K. F., HAYES, J. F., 1982: Effects of potassium dichromate and sample storage time on fat and protein by Milko-Scan and on protein and casein by a modified Pro-Milk Mk II method. Journal of Dairy Science, 65, 1895-1899.

SATO, T., IWAMOTO, M., HASHIZUME, K., YOSHINO, M., FURUKAWA, S., SOMEYA, Y., YANO, N., 1985: Near infrared spectrophotometric analysis for measuring major constituents of raw milk. Japanese Journal of Zootechnical Science, 56, 11, 878-882.

SATO, T., YOSHINO, M., FURUKAWA, S., SOMEYA, Y., YANO, N., UOZUMI, J., IWAMOTO, M., 1987: Analysis of milk constituents by the near infrared spectrophotometric method. Japanese Journal of Zootechnical Science, 58, 8, 698-706.

SJAUNJA, L. O., 1984 a: Studies on milk analysis of individual cow milk samples. I. Infrared spectrophotometry for analysis of fat, protein and lactose in milk. Acta agriculturae scandinavica, 34, 249-259.

SJAUNJA, L. O., 1984 b: Studies on milk analysis of individual cow milk samples. II. Factors affecting milk analyses by infrared technique under laboratory conditions. Acta agriculturae scandinavica, 34, 260-272.

SJAUNJA, L. O., 1984 c: Studies on milk analysis of individual cow milk samples. III. The effect of dif- ferent treatments on infrared analyses. Acta agriculturae scandinavica, 34, 273-285.

SJAUNJA, L. O., PHILIPSSON, J., LUNDSTRÖM, K., SWENSSON, CH., 1984: Studies on milk analysis of individual cow milk samples. IV. Factors affecting milk analyses in a routine system by automated apparatuses. Acta agriculturae scandinavica, 34, 286-299.

SJAUNJA, L. O., ANDERSSON, I., 1985: Laboratory experiments with a new infrared (IR) milk analyzer, the Milko-Scan 605. Acta agriculturae scandinavica, 35, 345-352.

SZIJARTO, L. F., BARNUM, D. A., 1984: Preparation, use and evaluation of standards developed for simultaneous monitoring of Coulter and Fossomatic electronic cell counting instruments in Ontario. Journal of Food Protection, 47, 3, 227-231.

SZIJARTO, L. F., HARDING, F., HILL, A. R., MELICHERCIK, J., 1990: Cooling systems for transport of unpreserved milk samples. Journal of Dairy Science, 73, 2299-2308.

ŠUSTOVÁ, K., KUCHTÍK, J., KRÁČMAR, S., 2006: Analysis of ewe's milk by FT near infrared spectroscopy: measurement of samples on Petri dishes in reflectance mode. Acta universitatis agriculturae et silviculturae Mendelianae Brunensis (Brno), 53, 2, 131-138.

ŠUSTOVÁ, K., RŨŽIČKOVÁ, J., KUCHTÍK, J., 2007: Application of FT near spectroscopy for determination of true protein and casein in milk. Czech Journal of Animal Science, 52, 9, 284-291.

TOMÁŠKA, M., SUHREN, G., HANUŠ, O., WALTE, H. G., SLOTTOVÁ, A., HOFERICOVÁ, M., 2006: The application of flow cytometry in determining the bacteriological quality in raw sheep's milk in Slovakia. Lait, 86, 127-140.

TSENKOVA, R., ATANASSOVA, S., ITOH, K., OZAKI, Y., TOYODA, K., 2000: Near infrared spectroscopy for biomonitoring: Cow milk composition measurement in a spectral region from 1,100 to 2,400 nanometers. Journal of Animal Science, 78, 515-522.

VALENBERG VAN, H. J. F., 1990: Standardization and control of instruments for analysis of milk. International Dairy Federation Congress, Montreal, 1316-1321.

VINES, D. T., JENNY, B. F., WRIGHT, R. E., GRIMES, L. W., 1986: Variation in milk fat, protein and somatic cell count from four dairy herd improvement laboratories. Journal of Dairy Science, 69, 2219-2223.

VOORT VAN DE, F. R., KERMASHA, S., SMITH, J. P., MILLS, B. L., NG-KWAI-HANG, K. F., 1987: A study of the stability of record of performance milk samples for infrared milk analysis. Journal of Dairy Science, 70, 1515-1523. 
Address

Doc. Ing. Oto Hanuš, Ph.D., Radoslava Jedelská, Jaroslav Kopecký, Agrovýzkum Rapotín, Agriresearch Rapotín, Výzkumníků 267, 78813 Vikýřovice, Česká republika, Czech Republic, Ing. Pavel Hering, Zdeněk Motyčka jun., Českomoravská společnost chovatelů, a. s. Praha, Laboratoř pro rozbor mléka Buštěhrad, Czech Moravia Breeders Association (CMBA) Prague, Milk testing laboratory Buštěhrad, Lidická 2/334, 27343 Buštěhrad, Česká republika, Czech Republic, Ing. Václava Genčurová, Výzkumný ústav pro chov skotu Rapotín, Research Institute for Cattle Breeding Rapotín, Výzkumníků 267, 78813 Vikýřovice, Česká republika, Czech Republic 